\title{
Rasgos comparativos de programas de reenganche formativo y sociolaboral en el ámbito no formal en Gijón (Asturias)
}

\section{Comparative features in formative \& socio- labour re-engaging programs within non-formal educational contexts in Gijón (Asturias)}

\author{
Soraya Calvo González ${ }^{1}$ \\ calvosoraya@uniovi.es \\ Sue Gutiérrez Berciano \\ gutierrezsue@uniovi.es \\ Cristina Bayarri López \\ bayarricristina@uniovi.es \\ Universidad de Oviedo, España.
}

\section{Resumen:}

El abandono escolar, producto de un proceso de desenganche progresivo del alumnado a la institución escolar, continúa siendo uno de los ejes prioritarios de estudio e intervención en nuestro sistema educativo. También lo son las diversas trayectorias de reenganche formativo $y$ sociolaboral de jóvenes que abandonaron tempranamente los procesos educativos.

El presente artículo se enmarca dentro del proyecto nacional coordinado de $\mathrm{I}+\mathrm{D}+\mathrm{I}$ "Procesos de reenganche educativo y sociolaboral de adolescentes en situación de vulnerabilidad. Estudio de casos e implicaciones socioeducativas" [MINECO17-EDU2016-76306-C2-2-R]. Destacamos dos objetivos clave del proyecto. Por

\begin{abstract}
:
Early school leaving, the result of students' progressive disengagement with schools, is still one of the main axis of study and intervention in our education system. The different re-engaging training and sociolabour paths that might be designed for youths who dropout from schools are another important field of study and intervention.

This article is framed within a project funded by the Spanish Ministry of Education called "Educational and Socio-Labour Re-engaging Processes of Disadvantaged Young People. Case Studies and SocioEducational Consequences". Two objectives are key for our study. On the one hand, to define the characteristics and the
\end{abstract}

1 Dirección para correspondencia (correspondence address):

Soraya Calvo González. Universidad de Oviedo. Departamento de Ciencias de la Educación. Facultad de Formación del Profesorado y Educación. Edificio Norte. C/ Aniceto Sela, s/n. 33005 Oviedo (España). 
Rasgos comparativos de programas de reenganche formativo y sociolaboral en el ámbito no formal en Gijón (Asturias)

Soraya Calvo González, Sue Gutiérrez Berciano y Cristina Bayarri lópez

un lado, conocer características y contextos de implementación de programas de prevención del abandono y reenganche dirigidos a adolescentes en situación vulnerable que están obteniendo resultados positivos. Por el otro, analizar historias y trayectorias educativas resilientes de jóvenes participantes en programas de diversas instituciones socioeducativas que han logrado superar el proceso con éxito.

Este trabajo ofrece un análisis de los rasgos comparativos -organizativos, de estructura, pedagógicos e institucionales- de programas de reenganche exitosos desarrollados en entidades socioeducativas de Gijón (Asturias). Para ello planteamos un enfoque metodológico cualitativo basado en estudios de casos orientados a la descripción. En concreto, presentamos programas contextualizados en entornos diferenciados con el fin de plantear comparaciones. Tras la presentación de los datos trabajados se ofrecerá una discusión y reflexión final destinada a derivar implicaciones de interés para la revisión, mejora y visibilización de los programas exitosos en términos de reenganche.

\section{Palabras clave:}

Fracaso escolar; programas de reenganche; organizaciones no gubernamentales; joven y éxito. prevention contexts of early school leaving and the implementation of successful re-engaging programs for disadvantaged youths. And, on the other hand, to analyse resilient educational personal stories and trajectories of youths who have successfuIly participated in educational programs. This article provides an analysis of the comparative features -organizational, structural, pedagogical and institutionalof successful re-engaging programs developed in socio-educational institutions in Gijón (Asturias). To this end, we used a qualitative approach based on the description of case studies. More specifically, we compare different re-engaging programs implemented in different contexts. Following the reporting of the data, we present some final considerations from which lines to review, improve and raise awareness on re-engaging programs can be inferred.

\section{Key words:}

Early school leaving; re-engaging programs; NGO; youth and success.

\section{Résumé:}

Le décrochage scolaire, produit d'un processus de désengagement progressif des étudiants de l'institution scolaire, continue d'être l'un des axes prioritaires d'étude et d'intervention du système éducatif. De même que les différentes trajectoires de réengagement formatif et socio-professionnel des jeunes qui ont abandonné les processus éducatifs.

Cet article fait partie du projet national coordonné de $\mathrm{R}+\mathrm{D}+\mathrm{I}$ «Processus de réengagement éducatif et socio-professionnel des adolescents en situation de vulnérabilité. Études de cas et implications socio-éducatives »[MINECO-17-EDU2016-76306-C2-2-R]. Nous soulignons deux objectifs clés du projet. Tout d'abord, connaître les caractéristiques et les contextes de mise en œuvre des programmes de prévention de l'abandon et du réengagement destinés aux adolescents en situation de vulnérabilité qui obtiennent des résultats positifs. Et aussi, analyser des histoires et des trajectoires éducatives résilientes de jeunes participants à des programmes de diverses institutions socio-éducatives qui ont réussi à surmonter le processus.

Ce travail propose une analyse des caractéristiques comparatives -organisationnelles, structurelles, pédagogiques et institutionnelles- des programmes de réengagement réussis dans les entités socio-éducatives de Gijón (Asturias). Nous proposons une approche méthodologique qualitative basée sur des études de cas orientées vers la description. 
Plus précisément, nous présentons des programmes contextualisés dans des environnements différenciés afin de proposer des comparaisons. Après la présentation des faits, une discussion et une réflexion finales seront proposées afin de déduire les implications d'intérêt pour la révision, l'amélioration et la visibilité des programmes réussis en termes de réengagement.

\section{Mots clés:}

Échecscolaire; éducation; programmes de réengagement; jeune et réussite.

Fecha de recepción: 13-02-2020

Fecha de aceptación: 09-03-2020

\section{Planteamiento del problema objeto de estudio}

Este artículo tiene como objetivo profundizar en las características y condiciones de tres programas clave desarrollados en el marco de dos organizaciones de la ciudad de Gijón (Asturias) que desarrollan propuestas formativas de reenganche orientadas a jóvenes con itinerarios vitales complejos. A partir de las aportaciones teóricas derivadas de la investigación sobre el tema, se ha desarrollado un estudio de casos múltiples en cuatro comunidades autónomas (Asturias, Cantabria, Murcia y Valencia) que incluye dos unidades básicas de análisis relacionadas entre sí: por un lado, los programas desarrollados en sus dimensiones institucionales, organizativas y pedagógicas; por otro, las personas jóvenes que partiendo de circunstancias adversas han mostrado su capacidad de sobreponerse a ellas. Esta estrategia se enmarca en el proyecto nacional coordinado de I+D+I "Procesos de reenganche educativo y sociolaboral de adolescentes en situación de vulnerabilidad. Estudio de casos e implicaciones socioeducativas" ${ }^{2}$ que busca conocer en profundidad programas de reenganche para jóvenes en situación de vulnerabilidad que están obteniendo resultados positivos en términos de reinserción educativa y/o sociolaboral.

Gijón, con 274.159 habitantes (según el Padrón de Habitantes del Ayuntamiento de Gijón, a febrero de 2020)³, ha apostado históricamente

2 Proyecto correspondiente al Programa Estatal de Investigación, Desarrollo e Innovación orientado a los Retos de la Sociedad en el marco del Plan Estatal de Investigación Científica y Técnica y de Innovación [MINECO-17-EDU2016-76306-C2-2-R].

3 Dato de población oficial del Padrón de Habitantes actual del Ayuntamiento de Gijón (total por sexo; frecuencia de actualización diaria), consultado en https://transparencia.gijon.es/set/demografia/padron_total_sexo 
por la promoción educativa y cultural urbana. Desde los años 80, la capital del municipio se ha sumergido en un proceso de reconstrucción identitaria como un intento de minimizar los efectos de la reconversión naval-industrial. Esta apuesta por una nueva política municipal se ha materializado en diversos planes y programas de carácter urbanístico, sociolaboral y educativo, configurándose la Fundación Municipal de Cultura, Educación y Universidad como el organismo autónomo encargado de la gestión y organización de los servicios culturales y educativos del municipio. Todo ello unido a la conversión de Gijón en Ciudad Educadora a principios de siglo, integrándose en la Red de Ciudades Educadoras $^{4}$, internacionales y estatales, y diseñando su Proyecto Educativo de Ciudad en el curso 2001/02, vigente en la actualidad.

Es importante tener en cuenta alguno de los elementos socioculturales que configuran la vida en la ciudad/municipio. Según los últimos datos oficiales sobre la renta media (2016), los hogares del municipio disponen de media 29.000 euros anuales. No obstante, los datos evidencian una clara desigualdad económica intra distritos en la principal población del municipio. En Gijón residen unas 28.000 personas nacidas en el extranjero (según datos oficiales del Ayuntamiento, a febrero de 2020) ${ }^{5}$, un número notable en relación con la tasa poblacional total. Sin embargo, la pérdida poblacional se convierte en una inclinación demográfica del municipio: en Gijón hoy viven casi 6.000 ciudadanos y ciudadanas menos que hace diez años (INE, 2019). Tendencia extensible a los barrios donde desarrollan su actividad las entidades sociales de reenganche: el barrio de El Coto, ubicado en la zona Este de la ciudad con una población de más de 9.000 habitantes y con una renta próxima a la media municipal; y el barrio de La Calzada, ubicado en la zona Oeste, donde residen casi 25.000 ciudadanos/as y cuyos hogares tienen una de las rentas más bajas del municipio, de poco menos de 19.000 euros anuales.

4 La Asociación Internacional de Ciudades Educadoras (AICE) es una Asociación sin ánimo de lucro, constituida como una estructura permanente de colaboración entre los gobiernos locales comprometidos con la Carta de Ciudades Educadoras, que es la hoja de ruta de las ciudades que la componen. Las Redes de Ciudades Educadoras son las estructuras descentralizadas integradas por ciudades/ miembro de la AICE de un determinado territorio.

5 Dato de población oficial del Padrón de Habitantes actual del Ayuntamiento de Gijón (total habitantes nacidos en España y el extranjero por sexo; frecuencia de actualización diaria), consultado en https://transparencia.gijon.es/set/demografia/padron_total_habitantes. 
En este último barrio, uno de los más habitados del municipio, implementa su actividad la entidad Mar de Niebla desde el año 2004. El marcado carácter obrero del barrio unido a la distancia significativa que existe a la zona centro de la ciudad, dibujó un escenario identitario con sustantividad propia que orienta los proyectos y acciones de intervención comunitaria de la entidad. Estructura sus acciones socioeducativas en diferentes ámbitos de intervención: infancia, adolescencia y juventud; acción social; voluntariado y participación social y empleo-formación. En este último ámbito, se enmarca el programa La Ofi, foco de análisis en el proyecto de investigación.

Por otro lado, el marco estructural de la Agencia Local de Promoción Económica y Empleo, en el barrio de El Coto, se convierte en el escenario donde desarrollan sus acciones los otros dos programas de reenganche estudiados. Este organismo es un referente en materia de desarrollo local que aúna las políticas municipales formativas y sociolaborales ofertando diversos servicios entre los que se encuentran los programas seleccionados. Por un lado, las Escuelas de Segunda Oportunidad nacen en el contexto europeo en la década de los noventa como propuestas formativas alternativas, sumándose la escuela de Gijón a la Asociación Europea de Ciudades por las Escuelas de Segunda Oportunidad en el año 2002. Su actividad cotidiana se enmarca en la línea estratégica de prevención del abandono escolar en colaboración con centros de Educación Secundaria de la ciudad. La misión pedagógica de este recurso se fundamenta en el compromiso por la compensación educativa y en el acompañamiento personal, formativo y social de las personas jóvenes. Por otro lado, las Escuelas-Taller son proyectos de educación no formal reconocidos por la Administración estatal y autonómica como iniciativas promotoras de proyectos mixtos de formación y empleo. Son itinerarios singulares originados como respuesta a la situación de precariedad laboral y la alta tasa de desempleo juvenil ligado a un deterioro considerable del patrimonio cultural y del entorno urbano y/o medio ambiental. De este modo, este recurso se fundamenta desde sus inicios en una dinámica de formaciónaprendizaje-oficio con el objetivo de mejorar la ocupabilidad, formación y habilidades sociales de personas jóvenes desde un clima motivador. 


\section{Antecedentes y fundamentación teórica}

El abandono escolar temprano como fenómeno educativo continúa siendo una de las preocupaciones prioritarias europeas y estatales, cuya tasa todavía continúa siendo notablemente elevada en nuestro país $(17,3 \%$, según los últimos datos de la Encuesta de Población Activa referidos al año 2019). Este indicador hace referencia al porcentaje de jóvenes entre 18 y 24 años que no han completado la educación secundaria de segunda etapa y que actualmente no está participando en ningún itinerario educativo o formativo (Ryanet al., 2014). Una realidad educativa que dibuja escenarios complejos desde planos formativos, culturales, políticos y económicos, cuyos factores desencadenantes, individuales, socioinstitucionales y sistémicos-estructurales (Kaye, D'Angelo, Ryan \& Lörin, 2017) han sido extensamente analizados (Archambault, Janosz, Fulli \& Pagani, 2009; Escudero y Rodríguez, 2011; Tarabini, 2016). Haciendo alusión a la estrategia global de lucha contra el abandono escolar temprano, identificamos una triple distinción de medidas de actuación a desarrollar según la clasificación europea: preventivas, de intervención y de compensación (Comisión Europea/EACEA/Eurydice/Cedefop, 2014).

Entendemos los procesos de reenganche formativo y sociolaboral como un fenómeno consustancial a las medidas compensatorias, siguiendo la terminología europea, alternativas y/o de segunda oportunidad. Medidas de retorno educativo para aquel alumnado muy desvinculado de lo escolar o que ha abandonado sus estudios, que no exclusivamente se desarrollan en contextos formales (González, 2017), tal y como sucede con los programas analizados.

Analizamos este reenganche desde una perspectiva holística (Kahu, 2013) que interrelacione la mirada ideográfica con la mirada estructural e institucional, en su vertiente organizativa y pedagógica. Planteamos una perspectiva analítica centrada en los factores endógenos y exógenos (Romero y Hernández, 2019) del reenganche que "focalice su mirada en los procesos que se escoden detrás de éstos" (Montes, 2016, p. 5). Esta perspectiva holística y procesual se vincula a un enfoque positivo e institucional (González, 2015) que frene los discursos y explicaciones de atribución del desenganche, fracaso y abandono escolar a fallos individuales del propio alumnado y de sus contextos sociales y familiares (Dei, 2003).En línea con esta perspectiva teórica partimos de un concepto de reenganche no fragmentado y emergente, 
integrado por diferentes dimensiones establecidas en un juego deductivo-inductivo, definitorias de los procesos de reenganche de los y las jóvenes: formativa, laboral, familiar, sociocomunitaria, de desarrollo personal y otras transversales a las planteadas (resiliencia, equidad, género y bienestar, entre otras).

En el presente artículo ponemos el foco en las instituciones y en sus programas de reenganche en un municipio concreto de Asturias, entendiendo bajo qué condiciones y características desarrollan sus actuaciones concretas. Consideramos de este modo la entidad social (y sus programas específicos) como dimensión clave en la configuración de procesos de reenganche, siguiendo el mapa de dimensiones emergentes explicitado, que ejerce una notoria influencia en todas las dimensiones del reenganche planteadas más allá del plano formativo. La investigación actual sobre el tema de estudio plantea en esta línea la necesidad de sostener esta mirada de análisis multidimensional pues en las prácticas exitosas en términos de reenganche interfieren desde factores "curriculares, a otros más estructurales, relacionales, de clima y ambiente de aprendizaje, de apertura y relación con la comunidad" (González y San Fabián, 2018, p. 46).

Las instituciones socioeducativas no formales, alternativas, de segunda oportunidad, compensatorias o de reenganche (González, 2017) se convierten en instituciones-casa para un gran número de jóvenes que han abandonado los estudios desde situaciones vitales muy heterogéneas. Unos contextos que reenganchan desde un firme cuidado institucional (Borck, 2018) y una aceptación incondicional (Myconos, Thomas, Kimberley, Te Riele \& Swain, 2016) creando un sentido de comunidad basado en una ética de la aceptación del otro y otra con respeto, justicia y apreciación (Furman, 2002). Tales programas beben de unos valores institucionales sólidos, caracterizados además por generar espacios de reconstrucción de narrativas personales y proyectos vitales de futuro que integren positivamente las adversidades (Forés y Grané, 2012). En estos escenarios se reestructuran las creencias que las personas jóvenes tienen interiorizadas en torno a su decisión de abandonar los estudios para que puedan ejercer una influencia positiva en su propio reenganche (Nieto, Portela, Torres y Rodríguez, 2018). Programas y medidas que orientan una facilitación hacia y para la resiliencia de las personas jóvenes, entendida no ya como una cualidad individual sino como una "acción educativa comunitaria" (Ruiz-Román, Calderón-Almendros y Juárez, 
2017, p. 138) construida colectivamente dentro de los marcos de una entidad que busca transformar (se).

\section{Metodología}

El diseño se ha planteado como estudio de caso múltiple que enfatiza el carácter interpretativo y naturalista de la investigación cualitativa (Flick, 2014). Entendiendo caso, como unidad de análisis asociada al estudio de una organización (Steinar, 2013). Este método permite comprender el fenómeno objeto de estudio desde el contexto donde germina, contemplando los agentes protagonistas, estructuras y significados que los determinan. La particularidad del caso iluminará la comprensión del fenómeno en su globalidad, siendo transferibles, que no generalizables, los resultados alcanzados (Stake, 2005).

El propósito es conocer en profundidad la organización, sus profesionales y programas de reenganche educativo para jóvenes en situación de vulnerabilidad, por ello, es indispensable utilizar múltiples fuentes de evidencia. En primer lugar, la documentación de la entidad para abarcar la contextualización organizativa y pedagógica de las instituciones objeto de estudio (San Fabián, 2014). Y la entrevista en profundidad y semiestructurada, como técnica planteada para conocer el perfil y praxis profesional (Goodson, 2004).

Priorizar el carácter conversacional de las entrevistas para favorecer la inducción de categorías reseñables en la concepción holística del caso determinó la elaboración de un modelo básico de preguntas a aplicar por parte de las personas investigadoras-entrevistadoras. Interiorizar este esquema permitió conjugar y flexibilizar las preguntas a la hora de enfrentar la narración del interlocutor, favoreciendo la fluidez de la conversación. En este artículo se recogen principalmente las dimensiones: a) visión personal de la institución y programa (estructura, recursos, perfil de los destinatarios, política de admisión y de permanencia, valores y metas pedagógicas) y b) enfoque docente del formador (conocimiento de los usuarios, características de la intervención, estrategias didácticas, mecanismos y/o procesos de evaluación).

Una de las principales estrategias desarrolladas para validar la investigación cualitativa ha sido la triangulación, tanto de métodos, como de investigadores, teorías y datos (Simons, 2011). Por ello, se han con- 
templado y analizado: a) triangulación de teoría, aproximándonos a los datos desde posiciones diversas que confluían en el equipo, b) recuperación de información de diversas fuentes y c) participación de varios investigadores en la misma fase del proceso.

\section{Procedimiento}

Para llegar a estos informantes clave fue preciso un acuerdo entre las partes y una selección de tipo intencional, siendo las personas responsables de las entidades las encargadas de facilitar el contacto con profesionales implicados y significativos. Bajo la premisa de éxito en el programa se seleccionaron los casos de los profesionales participantes, teniendo en cuenta criterios como: solvencia técnica, compromiso y apego con los y las jóvenes participantes, así como otros factores que considerasen idóneos desde la entidad y que indicasen la naturaleza genuina de su desempeño.

Tabla 1.

Temporalización del proceso de investigación en las entidades de Gijón

\begin{tabular}{|c|c|c|c|}
\hline Fase de investigación & Fecha & Entidad & Informantes \\
\hline \multirow[t]{2}{*}{ Acuerdo } & Enero 2018 & $\begin{array}{l}\text { Agencia Local } \\
\text { de Empleo }\end{array}$ & $\begin{array}{l}\text { Director del área, } \\
\text { Jefe de Servicio y } \\
\text { Coordinadores/as }\end{array}$ \\
\hline & Marzo 2018 & Mar de Niebla & $\begin{array}{l}\text { Responsable Área } \\
\text { Educación }\end{array}$ \\
\hline \multirow{5}{*}{$\begin{array}{l}\text { Desarrollo de } \\
\text { entrevistas }\end{array}$} & Enero 2018 & \multirow{4}{*}{$\begin{array}{l}\text { Agencia Local } \\
\text { de Empleo }\end{array}$} & $\begin{array}{l}\text { Coordinador Escue- } \\
\text { la Taller }\end{array}$ \\
\hline & Febrero 2018 & & $\begin{array}{l}\text { Coordinadora } \\
\text { Escuela de Segunda }\end{array}$ \\
\hline & $\begin{array}{l}\text { Marzo/Mayo } \\
2018\end{array}$ & & $\begin{array}{l}\text { Oportunidad } \\
\text { Formadores Escuela } \\
\text { Taller }\end{array}$ \\
\hline & $\begin{array}{l}\text { Mayo/Junio } \\
2019\end{array}$ & & $\begin{array}{l}\text { Formadoras Escuela } \\
\text { de Segunda Oportu- } \\
\text { nidad }\end{array}$ \\
\hline & Junio/Julio 2018 & Mar de Niebla & Formadores/as \\
\hline
\end{tabular}

Fuente: elaboración propia.

Tras el acuerdo entre las partes se programa una primera reunión para establecer el compromiso y participación en el estudio, iniciando los 
primeros contactos y la pauta de acceso al campo. Esta primera toma de contacto, coordinada por la entidad referente, facilitó un primer análisis del contexto y sus peculiaridades organizativas, así como una concreción de la temporalización del proceso de investigación. Un proceso compartido y coordinado entre las partes, que ha favorecido y fortalecido el estudio.

\section{Análisis de datos}

Se aplicó un procedimiento en espiral siguiendo un proceso interactivo deductivo-inductivo, derivando categorías desde las investigaciones previas y desde el análisis de la información recogida a través de las diferentes estrategias. Un análisis de contenido (Gibbs, 2012) realizado a través de un método exhaustivo y sistemático de comparación constante con los datos y la teoría (Andréu, García-Nieto y Pérez, 2007; Taylor y Bodgan, 1987). Desde este juego deductivo-inductivo, se ha establecido un mapa de categorías analíticas que contribuye a dar respuesta a las siguientes preguntas: ¿Cuáles son las características más destacables de la entidad y del programa de reenganche educativo seleccionado?, ¿Cómo diseñan y desarrollan su intervención educativa?, ¿Qué características y condiciones de entidad y programa han favorecido el éxito de las intervenciones?

Se elaboró un informe sobre cada uno de los casos analizados partiendo de las categorías emergentes, poniendo el foco para la elaboración de este artículo en aquellas dimensiones relativas a los programas de reenganche (características organizativas, programáticas y metodológicas, actitudinales y de desempeño profesional). Posteriormente, se establecieron comparaciones e integración de resultados entre los diversos casos-programa analizados. Los resultados obtenidos son presentados en función de la entidad y programa con el objetivo de ofrecer una visión profunda de cada caso, señalando elementos relevantes de coincidencia o diferencia en el apartado de conclusiones.

Un rasgo definitorio del proceso seguido es la fuerte familiarización (Rapley, 2014) de las tres investigadoras con los datos de la investigación: tanto la recogida como el análisis contrastado de la información fue realizado de forma colaborativa. 


\section{Resultados y discusión}

Nos encontramos ante entidades que a pesar de pertenecer al mismo contexto y compartir enfoque formativo al tener su ámbito de acción en el marco no formal, presentan características muy diferenciadas.

La Fundación para la Acción Social Mar de Niebla es una entidad sin ánimo de lucro privada. Su estructura es diversificada y si bien depende de un Patronato que debe velar por la consecución de ciertos objetivos formales, económicos o de impacto; su organigrama no depende de manera específica de un marco administrativo externo o superior concreto. Esta situación favorece en cierta medida la adaptación de proyectos y programas a las necesidades concretas y puntuales del entorno; así como la generación de nuevas propuestas. Mar de Niebla es por tanto una entidad con un carácter más fluido, adaptable y cambiante en función de los movimientos sociales de su entorno. El presupuesto de la entidad no depende en exclusiva de una única fuente de financiación, existiendo inyecciones económicas derivadas de entidades privadas, públicas, convenios institucionales e incluso donaciones. Esto puede conllevar cierta inestabilidad económica en determinados momentos o para determinados fines; aunque la forma jurídica de fundación y la solvencia económica que requiere soluciona en parte esta situación.

La Ofi, programa de la entidad que analizaremos en profundidad, está orientado a un público de entre 16 y 30 años y tiene un marco de actuación previo y estructurado con diferentes actividades y sub-programas. A pesar de su esquema previo, no hemos detectado limitaciones para la puesta en marcha de nuevas actividades, itinerarios e incluso horarios. Encontramos una excepción a esta situación: los proyectos desarrollados por La Ofi pero que dependen de entidades públicas como los itinerarios Joven Ocúpate ${ }^{6}$, dependientes del Servicio Público de Empleo, son claros ejemplos de proyectos pautados que deben seguir una organización cerrada en toda su trazabilidad. Los sub-programas propios de La Ofi son La Escuelina; que ofrece apoyo escolar orientado a obtener titulación de ESO, Competencias Clave o acceso a Ciclos Formativos; y programas específicos de empleo-formación que varían (talleres técnicos, proyectos de aprendizaje-servicio, etc.). También se plantea un servicio

6 Una de las medidas de Garantía Juvenil que busca facilitar la inserción laboral de los jóvenes y mejorar su empleabilidad, desarrollando diferentes acciones formativas. Están orientadas a jóvenes sin el graduado de Educación Secundaria Obligatoria. 
de orientación laboral y tutorización individualizada. La participación es voluntaria y la metodología es flexible, orientada a las individualidades y adaptable. La evaluación del proceso es individualizada y existe una coordinación continuada entre los y las profesionales vinculados al programa. La relación con el grupo es estrecha y diaria, lo que permite que existan mecanismos informales de evaluación continua que son socializados semanalmente en una reunión de coordinación de profesionales. No son usuales las pruebas tipo examen ni los resultados se miden en base a las técnicas clásicas de la escuela ordinaria.

Los profesionales de La Ofi no responden a un único perfil, aunque es evidente la orientación formativa del personal hacia el ámbito socioeducativo. Dentro del programa existen profesionales con titulación en Psicología, Trabajo Social, Magisterio o TASOC, entre otras. Mar de Niebla favorece la estabilidad del personal, existiendo un equipo base al que se suman profesionales que ofrecen respuestas puntuales de duración determinada. El acceso al empleo es a través de ofertas laborales de tipo ordinario siguiendo la misma dinámica que pudiera funcionar en cualquier entidad privada. Se tiene en cuenta la formación y la experiencia profesional o de voluntariado en ámbitos relacionados y con colectivos de personas jóvenes o infancia.

En contraposición nos encontramos con la Agencia Local de Empleo; una organización pública dependiente del Ayuntamiento de Gijón y que presenta niveles jerárquicos que conjugan elementos políticos, institucionales y administrativos. Los programas planteados en la entidad requieren una planificación multinivel que complejiza la adaptación de elementos formales y la generación de nuevos o diferentes proyectos. El presupuesto de la entidad es relativamente estable y está vinculada a los presupuestos del Ayuntamiento de Gijón, definiendo una pauta económica clara en la que sostener iniciativas puestas en marcha y que ofrecen cierta sensación de estabilidad económica. Esta pauta puede depender de dinámicas políticas no siempre vinculadas a las necesidades reales del entorno. A pesar de que la entidad depende formalmente de los presupuestos del Ayuntamiento, la Agencia Local de Empleo de Gijón es una de las "entidades promotoras de proyectos de formación y empleo" reconocidas por la Administración del Estado y del Principado de Asturias y, como tal, recibe subvenciones y financiación de otros organismos como la Consejería de Empleo, Industria y Turismo de Asturias.

Respecto a los dos programas estudiados, plantearemos sus caracte- 
rísticas por separado. En primer lugar, abordaremos la estructuración de La Escuela de Segunda Oportunidad (en adelante E2O). La E2O se autodefine como un recurso socioeducativo de transición o lanzadera hacia otros recursos formales de ámbito laboral, social o educativo. Se dirige a jóvenes de entre 14 y 25 años residentes en Gijón con dificultades para encajar en el sistema reglado y/o que han sufrido procesos de desenganche. La E2O aúna apoyo escolar orientado a la titulación de ESO, Competencias Clave o acceso a ciclos formativos y/o inserción sociolaboral desarrollando de manera equilibrada contenidos conceptuales y procedimentales. Junto a las actividades estructuradas a lo largo de las diferentes ediciones, la E2O participa de manera activa en actividades culturales, deportivas y lúdicas de la red socioeducativa de la ciudad; lo que plantea una clara conexión con el tejido comunitario y posibilidades reales de aprendizaje alternativo con enfoque lúdico. La participación es voluntaria y la metodología es participativa, estando muy presentes las tareas grupales. El proceso se enmarca en torno un proceso continuo de tutoría que incluye mecanismos de diagnóstico ecosistémico a partir de entrevistas individuales y acuerdos de compromiso en base a un Proyecto Individualizado con cierta flexibilidad. Tanto las dinámicas de intervención como las de evaluación están protocolizadas y se vinculan a una serie de estrategias previamente pautadas por parte de la coordinación estable del proyecto, la cual se reúne de manera periódica con los y las profesionales. Existen pruebas teórico-prácticas para comprobar la evaluación de los aprendizajes.

Los profesionales que participan en la $\mathrm{E} 2 \mathrm{O}$ son beneficiarios de planes de empleo de primera experiencia laboral promovidos por el Ayuntamiento de Gijón. Estos planes de empleo ofrecen puestos temporales (generalmente de un año) a recién egresados/as en ciclos formativos y/o titulaciones universitarias del ámbito socioeducativo. Las personas que acceden a los puestos ofertados asumen roles de maestro/a y monitores/ as, pero no suelen tener experiencia laboral previa ni en temas de abandono escolar o reenganche ni con el colectivo destinatario de la E2O. La valoración de perfiles se hace en función de los criterios planteados en las bases de los planes de empleo, y suelen presentar limitaciones de acceso en función de edad, fecha en la que se obtuvo el título o participación previa en otras ediciones. Esta situación conlleva una inestabilidad en la plantilla vinculada a la E2O ya que si bien existe una estructura de base y coordinadora de todo el proceso que sí es estable (personal del 
Ayuntamiento de Gijón en régimen laboral o funcionario), la práctica totalidad de profesionales que llevan a cabo labores docentes, de monitorización y tutorización encaja en este modelo temporal. Por otro lado, la entidad no facilita procesos de capacitación continua; por lo que la especialización formativa es completamente autónoma.

El otro programa analizado dependiente de la Agencia Local de Empleo es el de las Escuelas Taller (en adelante ET). Cada ET consiste en un itinerario de empleo y formación para jóvenes desempleados/as no ocupados e inscritos en el Servicio Público de Empleo de entre 16 y 25 años. Cuentan con financiación del Fondo Social Europeo, del Servicio Público de Empleo del Principado de Asturias (SEPE) y del Ayuntamiento de Gijón. Son proyectos de largo recorrido en nuestro país y en las últimas décadas tanto la organización como las condiciones de acceso se endurecen y pautan para responder a la normativa del Espacio Económico Europeo y su correspondiente reconocimiento formativo, desde el SEPE y el Principado de Asturias. Las ET han sufrido varios cambios en los últimos años ya que se han tenido que amoldar a los requerimientos europeos de los certificados de profesionalidad. Cada ET se enfoca en una profesión/temática específica, de manera que tras la superación del proceso formativo el alumnado obtiene una titulación acreditada y ajustada a los parámetros de los certificados de profesionalidad (de tipo 1, 2 y 3) que le permite acceder a determinados puestos de trabajo. La duración de este tipo de programas es de entre 1 y 2 años con una estructura prefijada en la que podemos distinguir dos fases: la primera, de 6 meses de duración, destinada a la formación profesional teórico-práctica y en la que el alumnado percibe una ayuda económica en forma de "beca"; y la segunda, que dura de 6 a 18 meses, en la que se firma un contrato con la entidad promotora y se percibe el $75 \%$ del SMI vigente cada año mientras se realiza un trabajo de utilidad pública. La metodología de trabajo de las ET permite la adaptación al grupo, pero debe responder a las exigencias de los certificados de profesionalidad en los que se asienta el programa. Debido a la diferenciación por fases, la formación está claramente estructurada en teoría (priorizada durante la primera fase, en aula) y práctica (priorizada durante la segunda fase, en campo u obra) y la evaluación debe ajustarse también a distintas normas protocolizadas que garantizan el cumplimiento de los objetivos básicos. Aunque la finalidad de este tipo de programas es la inserción laboral de las personas participantes, durante todo el proceso teórico-práctico es relevante el 
trabajo en torno a cuestiones generalistas y relacionadas con competencias sociolaborales como hábitos de comportamiento, bienestar y salud, manejo informático, etc. La tutorización es continua durante el proceso de formación en la Escuela y, también en el momento de acceso al entorno laboral: en todo momento se busca el hilo conductor del proceso educativo que apoye el reenganche gracias a un acompañamiento.

El personal que conforma las ET carece de estabilidad debido al sistema en el que engrana la contratación de profesionales. Cada edición suele ir acompañada de una convocatoria pública de empleo temporal en el que se valoran méritos, experiencia y formación vinculada al puesto, pero que en cada ocasión suele empezar "desde cero". De esta manera el personal que participó en anteriores ediciones debe volver a presentarse a la nueva convocatoria perdiendo antigüedad y encadenando contratos. Generalmente, el personal de las ET está muy formado en el puesto gracias a una experiencia dilatada muy ligada a programas de empleo-formación (ya sea en anteriores ET, en las antiguas Casas de Oficios ${ }^{7} \mathrm{O}$ en las nuevas fórmulas como Joven Ocúpate). Al no existir formación oficial reglada en relación con estos escenarios tan concretos, la adecuación profesional viene o por la experiencia profesional o por la formación continuada en temáticas sociolaborales, socioeducativas, etc. Las ET presentan dos perfiles clave que deben trabajar de manera coordinada: Maestros/as y maestros/as de oficio. Los/as maestros/as orientan y pautan contenidos específicos más bien teóricos para la obtención de las titulaciones oficiales vinculadas al itinerario formativo; y los/as maestros de oficio trabajan contenidos técnicos y experimentales de adecuación al puesto de trabajo gracias a las unidades de competencia propias del certificado profesional objetivo del programa.

\section{Conclusiones}

Tras desgranar tres programas de dos entidades de tradición en Gijón/ Xixón con elementos pedagógicos y estructurales claramente diversos, creemos conveniente resaltar algunas ideas relevantes.

7 Las Casas de Oficios son proyectos de carácter temporal en los que el aprendizaje y la cualificación se alternan con un trabajo productivo en actividades de utilidad pública o social que permita la inserción a través de la profesionalización y adquisición de experiencia de los participantes. Siguen una estructura similar a las Escuelas Taller pero tienen algunas diferencias formales. 
En primer lugar, resulta evidente la mayor flexibilidad didáctica y de enfoque que Mar de Niebla presenta frente a la Agencia Local de Empleo. Esto es debido, principalmente, a su naturaleza de entidad del tercer sector, menos burocratizada que en el caso del servicio municipal. A pesar de que existen plazos, subvenciones y convenios que la Fundación debe asumir, parece constatada la tendencia elástica que sus programas presentan, especialmente aquellos que no dependen de convocatorias externas pautadas por organismos estatales o autonómicos. Esto puede ser un elemento efectivo si entendemos que los programas de reenganche son herramientas que deben superar el marco cerrado y formal que se plantea en el sistema reglado ordinario. Sin embargo, es destacable que, si bien en esta ocasión aparece como un rasgo positivo de la entidad, también puede complejizar el trabajo diario en una organización que no tenga buena cohesión grupal o que carezca de un foco definido aceptado por todos sus miembros. En ese caso, la estructura prefijada y protocolizada de los programas municipales podría convertirse en un anclaje necesario en el que apoyar acciones formativas con un mínimo de garantías en cuanto a pautas de acción.

En segundo lugar, es reseñable la pluralidad de perfiles profesionales que encontramos. A pesar de esa pluralidad existe una doble vertiente común: profesionales de lo socioeducativo y profesionales de cuerpos técnicos y/o expertos en materias específicas. La conjunción de ambos perfiles parece convertirse en un engranaje necesario para que estos programas funcionen de manera satisfactoria. La unión de teoría y práctica, sin dejar de lado la necesidad de obtener titulaciones capacitantes como la ESO o las cualificaciones profesionales, permite llevar a la concreción aquellas cuestiones que a priori pueden resultar abstractas. El foco en la praxis es una de las diferencias fundamentales que estos programas plantean respecto a la enseñanza tradicional y vinculada con el marco escolar formal.

En tercer lugar, resulta llamativo que la plantilla profesional en la que más estabilidad encontramos sea precisamente la que a priori puede parecer más inestable debido al carácter "informal" de la entidad que la sostiene. La inestabilidad es una constante en los programas vinculados a la entidad pública, pudiendo ser traducida en peores condiciones de vida para las personas trabajadoras y en dificultades para generar sentimiento de grupo entre profesionales y/o canalizar propuestas de innovación y mejora para futuras ediciones del proyecto. La incorpora- 
ción continuada de nuevas personas a unos programas con objetivos tan concretos marcas dinámicas de inicio-fin tanto en los propios itinerarios formativos como en las vinculaciones del alumnado con sus referentes de orientación y reenganche.

A modo de resumen de características comunes, todos los casos estudiados se adecuan a tramos de edad concretos que se sitúan en la juventud y que se inician en la adolescencia temprana. También encontramos en todos los programas un interés por garantizar la titulación en ESO de los y las participantes; así como el trabajo en torno a habilidades complementarias y orientadas a una mejora de la calidad de vida laboral y personal. Así mismo, todos los programas ofrecen una tutorización continuada, con tendencia a la individualización, y con una metodología de intervención flexible y adaptable. En algunos casos, como la E2O o acciones propias de La Ofi, esto aparece más claramente reflejado que en otros, como los programas de empleo formación de las ET o los Joven ocúpate. Sin embargo, el acompañamiento continuado y la adecuación a las diversidades personales parecen rasgos determinantes y comunes de este tipo de programas de reenganche (González, 2017; González y San Fabián, 2018; McGregor y Mills, 2011; Myconos et. al., 2016). Otro rasgo común es la fuerte coordinación necesaria y detectada entre profesionales y que puede verse reflejada en todos los casos estudiados.

Como crítica y previsión de un futuro problema, nos gustaría poner el foco en dos situaciones destacables. Por un lado, la adecuación de itinerarios de Escuelas Taller a certificados de profesionalidad de nivel 2 y 3 frente a los de nivel 1, no estableciendo estos últimos requisitos específicos de acceso. Si atendemos al colectivo general al que los programas de reenganche formativo y sociolaboral suelen orientarse podemos contrastar (Montes, 2016; Prieto, 2015; Tarabini, 2016) como una parte significativa de las personas que acceden a estos programas carecen de cualquier tipo de titulación oficial: son jóvenes que mayoritariamente han abandonado el sistema educativo reglado antes de la finalización de la edad de escolarización obligatoria o que han completado el proceso pero sin obtener el título de Graduado en Educación Secundaria Obligatoria. En este punto estaríamos hablando de fracaso escolar, y parece evidente que sin resolver este problema "será prácticamente inviable avanzar" (Escudero y Rodríguez, 2011, p. 1) hacia el abordaje del abandono escolar temprano.

Las personas verdaderamente desenganchadas del sistema escolar 
formal que han abandonado sus estudios requieren acciones formativas básicas que se encaminen a la obtención de certificados de nivel 1. Esto puede derivar en una perversión de la idea inicial de este tipo de programas; orientando los esfuerzos a un público con más posibilidades formativas. Por el otro lado, la escasez de formaciones regladas u oficiales que traten de dar respuesta al trabajo con este colectivo concreto y con unas necesidades tan significativas. Vemos que el personal que trabaja en estos entornos viene en su mayoría de titulaciones relacionadas con la educación, la psicología o el mundo de lo social. Creemos que podría ser interesante plantear una vía formativa concreta para atender a las necesidades de los profesionales que trabajan en programas de reenganche que, a su vez, consiga dotar de valor su trabajo ya que en muchas ocasiones se sienten "en tierra de nadie". La generación de un plan formativo formal podría abrir vías necesarias para la profesionalización de este personal, lo cual reinvertiría de manera multinivel: en la formación recibida por parte del alumnado, en la valorización social del trabajo realizado, y en la estabilización de unos programas que tratan de dar solución a problemas sociales generados por una estructura educativa y laboral rígida que deja a mucha gente al margen.

\section{Limitaciones del estudio}

Sincronizar los tiempos de investigación con las entidades participantes se hizo complicado, y en algún caso, se dilató más de lo esperado, pero los imprevistos por ambas partes están a la orden del día. El contacto directo con las entidades y el vínculo establecido, ofrecieron la comprensión de esta coyuntura particular.

La investigación ha sido definida para la muestra de programas especificados, y si bien nuestras conclusiones no pueden ser generalizables debido al carácter localista de los datos presentados sí posibilitan la reflexión sobre tendencias que pueden ser transferibles a contextos similares. En este sentido, existe escasa producción científica de estudios sobre reenganche formativo y/o sociolaboral en el ámbito no formal, pero las posibilidades de estudio han sido planteadas y deben servir de aliciente para continuar con esta línea de investigación. 
Rasgos comparativos de programas de reenganche formativo y sociolaboral en el ámbito no formal en Gijón (Asturias) Soraya Calvo González, Sue Gutiérrez Berciano y Cristina Bayarri lópez

\section{Referencias}

Andréu, J.; García-Nieto, A. y Pérez, A.M. (2007). Evolución de la teoría fundamentada como técnica de análisis cualitativo. Madrid: Centro de Investigaciones Sociológicas.

Archambault, I., Janosz, M., Fulli, J. \& Pagani, L. (2009). Student Engagement and its Relationship with Early High School I Dropout. Journal of School Health, 3(32), 651670.

Borck, C.R. (2018). "College Material" Structural Care at a New York City Transfer School. Societies, 7, 1-13.

Comisión Europea/EACEA/Eurydice/Cedefop (2014). Tackling Early Leaving from Education and Training in Europe: Strategies, Policies and Measures. Eurydice and Cedefop Report. Luxembourg: Publications Office of the European Union.

Dei, S. (2003). Schooling and the dilemma of youth disengagement. McGill Journal of Education, 38(2), 241-256.

Escudero, J.M. y Rodríguez Entrena, M. J. (2011). Afinar la comprensión y movilizar políticas consecuentes para afrontar el abandono escolar temprano, y algo más. Avances de Supervisión Educativa, 14, 1-15.

Flick, U. (2014). El diseño de la Investigación Cualitativa. Madrid: Morata.

Forés, A. y Grané, J. (2012). La resiliencia en entornos socioeducativos. Madrid: Editorial Narcea.

Furman, G. (2002). School as community: from promise to practice. EEUU: SUNY series.

Gibbs, G. (2012). El análisis de datos cualitativos en Investigación Cualitativa. Madrid: Morata.

Goodson, I. F. (2004). Historias de vida del profesorado. Barcelona: Octaedro.

González, M.T. (2015). Los centros escolares y su contribución a paliar el desenganche y abandono escolar. Profesorado: Revista de Curriculum y Formación del Profesorado, 19(3), 158-176.

González, M.T. (2017). Desenganche y abandono escolar, y medidas de reenganche: algunas consideraciones. Profesorado. Revista de Curriculum y Formación del Profesorado, 21(4), 17-37.

González, M.T. y San Fabián, J.L. (2018). Buenas prácticas en medidas y programas para jóvenes desenganchados de lo escolar. Revista Iberoamericana sobre Calidad, Eficacia y Cambio en Educación, 16(1), 41-60.

Kahu, E.R. (2011). Framing student engagement in higher education. Studies in Higher Education, 38(5), 758-773.

Kaye, N., D’Angelo, A., Ryan, L. \& Lőrin, M. (2017). Early School Leaving: Risk and Protective Factors. RESL.eu Publications, 4, 1-59.

McGregor, G. y Mills, M. (2011). Alternative education sites and marginalised young people: 'I wish there were more schools like this one'. International Journal of Inclusive Education, 16(8), 843-862.

Montes, A. (2016). En la encrucijada. Factores de desenganche y reenganche educativo en la trayectoria de seis jóvenes en Cataluña (España). INGURUAK Revista Vasca de Sociología y Ciencia Política, 61, 1-22.

Myconos, G., Thomas, J., Kimberley, W. y Te Riele, K. \& Swain, L. (2016). Educational 
Rasgos comparativos de programas de reenganche formativo y sociolaboral en el ámbito no formal en Gijón (Asturias)

Soraya Calvo González, Sue Gutiérrez Berciano y Cristina Bayarri lópez

re-engagement as social inclusion: the role of flexible learning options in alternativeprovision in Australia. FORUM, 58(3), 345-354.

Nieto Cano, J.M., Portela Pruaño, A., Torres Soto, A. y Rodríguez Entrena, M.J. (2018). Del abandono educativo temprano al reenganche formativo: un estudio narrativo con biogramas. Educatio Siglo XXI, 36(2) 93-114.

Prieto, B. (2015). El camino desde la vulnerabilidad escolar hacia el desenganche educativo. El papel de las escuelas de segunda oportunidad en la estrategia contra el abandono educativo. Profesorado. Revista de Curriculum y Formación de Profesorado, 19(3), 110-125.

Rapley, T. (2014). Los análisis de la conversación, del discurso y de documentos en Investigación Cualitativa. Madrid: Ediciones Morata.

Romero Sánchez, E. y Hernández Pedreño, M. (2019). Análisis de las causas endógenas y exógenas del abandono escolar temprano: una investigación cualitativa. Educatio Siglo XX1, 22(1), 263-293.

Ruiz-Román, C., Calderón-Almendros, I. \& Juárez, J. (2017). La resiliencia como forma de resistir a la exclusión social: un análisis comparativo de casos. Pedagogía Social. Revista Interuniversitaria, 29, 129-141.

Ryan, L., Lorinc, M., D'Angelo, A., Kaye, N., Araujo, H., Magalhaes, A., Rocha, C., Macedo, E., Szalai, J. \& Kende, A. (2014). Policies on early school leaving in nine European countries: a comparative analysis. Budapest, Hungary: Centre for Policy Studies.

San Fabián, J.L. (2014). Evaluar programas socioeducativos en tiempos de crisis: un enfoque organizacional. Oviedo: Trabe.

Simons, H. (2011). El estudio de caso: Teoría y práctica. Madrid: Morata.

Stake, R. E. (2005). Investigación con estudio de casos. Madrid: Morata.

Steinar, K. (2013). Las entrevistas en investigación cualitativa. Madrid: Morata.

Tarabini, A. (2016). La exclusión desde dentro o la persistencia de los factores "push" en la explicación del abandono escolar prematuro. Organización y gestión educativa: Revista del Fórum Europeo de Administradores de la Educación, 24(3), 8-12.

Taylor, S.J. y Bodgan, R. (1987). Introducción a los métodos cualitativos de investigación. Barcelona: Ediciones Paidós Ibérica. 